\title{
The French Health Care System and its Reform
}

\author{
Shinya MATSUDA*1, Yutaka MOTOHASHI*2 \\ * Department of Preventive Medicine and Community Health, University of Occupational and \\ Environmental Health, Kitakyushu \\ ${ }^{*}$ Department of Public health, School of Medicine, Akita University, Akita
}

\begin{abstract}
The French health care system is characterized by its social insurance scheme with universal coverage, freedom of prescription and of location by the doctor, fee-for-service payment by patients, and free choice of doctor by patients, which essentially tends to inflate its health expenditures. Thus, the health expenditures of France has been increasing over the average expenditures of other European countries. Under the principle of autonomy of the social insurance fund, the increase in medical expenditures has been absorbed by a decrease in the reimbursement rate and an increase in the contribution rate of the insured. However, it is no longer possible to cope with this financial crisis by these traditional measures because of the worsening of the unemployment problem. Nowadays, the increase in the contribution rate is regarded as one of the important reasons for the economic stagnation of the French society. In order to cope with this difficult situation, the French government has changed its health policy from the demand side strategy to the supply side strategy. The concrete plan of this policy was presented by Prime Minister Alain Juppé (the Juppé plan). The plan consists of; 1) unification of the medical insurance scheme, 2) establishment of a ceiling on medical expenditures, 3) regionalization of health policy, 4) disclosure of medical information, 5) introduction of medical references, 6) creation of a social protection scheme for the dependent elderly, 7) introduction of an object tax for the social security fund, etc.. These subjects have been materializing step by step after many twists and turn. The most important principles of the plan are the transparency of the medical information and the responsibility of each actor within the health system. The French government has conducted a lot of international comparable studies of health systems. According to the results of a series of active and profound discussions, the French government has conducted various social experiments. It is very meaningful for the Japanese government to analyze this process of health reform occurring in France in order to discuss the health system reforms in Japan.
\end{abstract}

Jpn J Hyg 1998; 53: 511-20

\section{Present and Future Cancer Risk Assessment of Carcinogenic Heterocyclic Amines in Humans}

\author{
Shigeo MANABE, Hiroko MATSUSHITA \\ *'Department of Hygiene and Preventive Medicine, University of Tokyo, Tokyo. \\ ${ }^{* 2}$ College of Health Professions, Toho University, Tokyo.
}

\begin{abstract}
Heterocyclic amines are formed during high temperature cooking of foods such as meat and fish. Although heterocyclic amines are known mutagens and some of them are shown to be carcinogenic to experimental animals, the carcinogenic potential of heterocyclic amines in humans has yet to be established. The most important criteria in determining the relationship between heterocyclic amines and human cancers are the strength and consistency of the association, the presence of a dose-response relationship, and the biological plausibility of heterocyclic amines being human carcinogens. To date, there are too few data to evaluate the strength, consistency, and dose response of this relationship in humans. To investigate cancer risk posed by heterocyclic amines to humans, an accurate estimation of exposure is needed. Validated methods for exposure assessment of heterocyclic amines are not available. At present, without any available long-term biomarkers of heterocyclic amines, it is crucial to improve exposure assessment. Most studies concerning the cancer risk assessment of heterocyclic amines are based on the exposure levels calculated only by information on the consumption of cooked foods and the mean levels of heterocyclic amines in cooked foods. Inaccurate exposure assessment can lead to incorrect cancer risk. This review describes the present and future problems of the exposure assessment and cancer risk assessment of heterocyclic amines.
\end{abstract}

Jpn J Hyg 1998; 53: 521-8 


\title{
Geographication of Centenarians and its Related Factors in Japan
}

\author{
*'Kazushi OKAMOTO, ${ }^{* 2}$ Kiyoko YAGYU \\ *' Department of Public Health, Aichi Prefectural College of Nursing and Health \\ *2 Department of Public Health, Aichi Medical University, Nagoya
}

\begin{abstract}
This study explores the factors associated with longevity in Japan by considering the geographic distribution of centenarians based on the 1990 population census.

The proportion of centenarians was calculated based on the population of those aged 65 years or older. The findings were 11.0 males and 28.2 females per 100,000 people aged 65 years or older. In this analysis, the rate ratio, RR, was used as an index for fluctuation of centenarians by prefecture. RR is also the proportion of centenarians in a prefecture to that in the nation. When the RR of both sexes is over 100, the region is defined as high proportion and less than this as low proportion. The high proportion regions were located in the western part of Japan i.e. from the Chugoku to the Kyusyu district, the low proportion regions were located in the eastern part of Japan i.e. from the Tohoku to the Kanto district both seres. The relationship between the geographical distribution of centenarians and environmental factors was analyzed. The correlation coefficient of the proportion of centenarians and the temperature as index of a regional difference was the highest among the factors investigated.

After adjusting for mean temperature, the mean value of factors associated with a high quality of welfare work and of medical service, and that of having leisure time were significantly higher in high proportion regions than in low proportion regions.
\end{abstract}

Jpn J Hyg 1998; 53: 529-35

\section{The Effects of Interferon- $a$ on Oxygen Radical Production by Human Neutrophils}

\author{
Takuya KOIE, Katsuhiko SUZUKI, Satoru KUDO, Mutsuo YAMADA, Qiang LIU, \\ Shigeyuki NAKAJI and Kazuo SUGAWARA
}

Department of Hygiene, Hirosaki University School of Medicine,Aomori

\begin{abstract}
This study was done to determine if the production and metabolism of reactive oxygen species from human neutrophils were modulated by the treatment of interferon- $\alpha$ (IFN- $\alpha$ ).

Luminol-dependent Chemiluminescence $(\mathrm{LmCL}$ ) responses were inhibited by a high concentration of IFN- $\alpha$ (more than $1 \times 10^{4} \mathrm{IU} / \mathrm{ml}$ ) when opsonized zymosan $(\mathrm{OZ})$ and phorbol 12-myristate 13-acetate(PMA) were used as stimulants. However, these responses were increased by $1 \times 10^{3} \mathrm{IU} / \mathrm{ml}$ of IFN- $\alpha$ with $\mathrm{Ca}^{2+}$-ionophore $\mathrm{A23187}$ stimulation.Lucigenin-dependent Chemiluminescence $\left(\mathrm{L}_{\mathbf{g}} \mathrm{CL}\right)$ responses were inhibited by all concentrations. These findinds suggest the possibility that IFN- $\alpha$ inhibits activation of protein kinase C(PKC), whereas the resultig effect might be due to the inhibition of myeloperoxidese(MPO) degranulation.

Preincubation of human neutrophils with IFN- $\alpha$ for 30,60 or 120 minutes and subsequent stimulation with OZ,PMA and $\mathrm{Ca}^{2+}$-ionophore $\mathrm{A} 23187$ caused an increase $\mathrm{LgCl}$ responses, while inhibiting $\mathrm{LmCL}$ responses. These findings suggest that preincubation of human neutrophils with a high concentration of IFN- $\alpha$ might enhance the NADPH-oxidase activity, although a relative increase of $\mathrm{LgCL}$ was due to the inhibition MPO degranulation.
\end{abstract}

Jpn J Hyg 1998; 53: 536-44 


\title{
A Follow-up Study on Renal Tubular Dysfunction in Women Living in the Cadmium-polluted Jinzu River Basin in Toyama, Japan \\ Part 1. Changes in the level of exposure to cadmium after soil replacement of polluted paddy fields and the related effects on the prognosis of renal tubular dysfunction
}

\author{
JianJun FAN, Keiko AOSHIMA, Terutaka KATOH, Hidetoyo TERANISHI and Minoru KASUYA
}

Department of Public Health, Faculty of Medicine, Toyama Medical and Pharmaceutical University, Toyama

\begin{abstract}
A follow-up study on renal tubular dysfunction was carried out on 193 female inhabitants of the cadmium (Cd)-polluted Jinzu River basin and 40 reference subjects living in an adjacent area in 1994-95. They were 54 to 70 years old when the initial examination was conducted in 1983-84. In the Cd-polluted Jinzu River basin, extensive reclamation of polluted rice fields has been conducted since 1979; as a result, the average $\mathrm{Cd}$ concentrations in polished rice consumed by the subjects in the 1994-95 study $(0.12 \mathrm{ppm}$ in 1994, $0.14 \mathrm{ppm}$ in 1995) were significantly lower than those in the $1983-84$ study $(0.26 \mathrm{ppm}$ in $1983,0.29 \mathrm{ppm}$ in 1984). The average $\mathrm{Cd}$ levels in urine in the follow-up study $(7.5 \mu \mathrm{g} / \mathrm{g} \mathrm{Cr}$. in $1994,7.7 \mu \mathrm{g} / \mathrm{g} \mathrm{Cr}$. in 1995) were also significantly lower than those in the initial study $(13.5 \mu \mathrm{g} / \mathrm{g}$ Cr. in $1983,13.3 \mu \mathrm{g} / \mathrm{g}$ Cr. in 1984). However, the mean values for urinary excretion of $\beta_{2}$-microglobulin $\left(\beta_{2}\right.$-m) $(3.9 \mathrm{mg} / \mathrm{g} \mathrm{Cr}$. in 1994, $3.7 \mathrm{mg} / \mathrm{g}$ Cr. in 1995) and glucose $(203 \mathrm{mg} / \mathrm{g} \mathrm{Cr}$. in $1994,251 \mathrm{mg} / \mathrm{g} \mathrm{Cr}$. in 1995) in the follow-up study were significantly higher than those obtained at the initial examination $(2.0 \mathrm{mg} / \mathrm{g} \mathrm{Cr}$. and $125 \mathrm{mg} / \mathrm{g} \mathrm{Cr}$. in 1983 and $1.1 \mathrm{mg} / \mathrm{g} \mathrm{Cr}$. and $78 \mathrm{mg} / \mathrm{g} \mathrm{Cr}$. in 1984 for $\beta_{2}-\mathrm{m}$ and glucose excretion, respectively). The magnitude of increase in urinary excretion of $\beta_{2}-\mathrm{m}$ and glucose in inhabitants of the $\mathrm{Cd}$-polluted area was significantly higher than that of the inhabitants of the reference area. Moreover, an increase was observed in the prevalence of renal tubular dysfunction determined by urinary $\beta_{2}-\mathrm{m}$ exceeding $10 \mathrm{mg} / \mathrm{g}$ creatinine and urinary glucose exceeding $150 \mathrm{mg} / \mathrm{g}$ creatinine only among inhabitants of the $\mathrm{Cd}$-polluted area; it is noteworthy that 31 new cases of renal tubular dysfunction were observed in the follow-up study. These results indicate that renal tubular dysfunction among inhabitants of the Cd-polluted Jinzu River basin is irreversible and progressive, and many new cases of renal tubular dysfunction were also noted over a period of 11 years, despite the fact that $\mathrm{Cd}$ exposure had decreased over the past 11 years.
\end{abstract}

Jpn J Hyg 1998; 53: 545-57 


\section{Environmental Health and Preventive Medicine Official Journal of the Japanese Society for Hygiene}

\section{INSTRUCTIONS FOR AUTHORS}

Environmental Health and Preventive Medicine as the official journal of the Japanese Society for Hygiene, is devoted to the publication of definitive studies including original papers, short communications and review articles, on human health sciences related to biological, physical, chemical, medical, psychosocial,and other various environmental factors.

\section{Categories of publication}

Submissions may include:

(1) Original papers reporting results of original fundamental research in any branch in the environmental health and preventive medicine field, should conform to the Journal style.

(2) Short Communications reporting on research considered to require speedy publication, receive rapid review. They should be no more than 4 printed pages in length, containing an Abstract of about 100 words, a one-paragraph introduction, an abbreviated Materials and Methods section, Results and Discussion sections (which may be combined), a maximum of 20 references, and no more than 5 items for the display of date (any combination of tables and illustrations).

(3) Reviews on subjects relevant of the field. Submission of an outline of an unsolicited review may be approved by the Editorial Board, after which the complete article will be carefully evaluated before acceptance.

(4) Announcements of scientific meetings and of courses in Environmental Health and Preventive Medicine should be submitted at least 1 month prior to issue.

\section{Editorial Policies}

Submissions must be accompanied with the authors written confirmation that research contained in the parer has not been and will not be submitted to or published in any other scientific journal before a decision has been made as to its acceptability for publication in Environmental Health and Preventive Medicine. All authors listed on a manuscript must have agreed to its submission and content, as determined in the Uniform Requirements for Manuscripts submitted to Biomedical Journals (JAMA1993;269;2282-6). Revisions to text or authors must be approved also by all authors. Environmental Health and Preventive Medicine accepts no responsibility for such changes. Associate Editors with broad expertise in all areas of Environmental Health and Preventive Medicine, along with a large group of external reviewers provide fair and thorough evaluation of papers submitted to Environmental Health and Preventive Medicine. Associate Editors and reviewers are expected to maintain a strict ethical code regarding confidentiality of the material under review. Any relation ships that could cause a conflict of interest with regard to a manuscript must by revealed by authors, reviewers, and Associate Editors to the Editor-inChief. After review, authors will be promptly informed of Editorial decision. Only written inquires regarding the status of manuscripts will be answered. Manuscripts only will be returned in the case of rejection. Accepted submissions become the property of the Japanese Society for Hygiene.

\section{Preparation of Manuscripts}

Original manuscripts must include:

(1) The author's covering letter containing the information in dupilate.

(2) Two copies of the manuscript and two sets of original illustrations. One set of original illustrations should be designated for use by the printer.

Mailing Address

Manuscripts should be sent to the Editorial Office,

clo Department of Preventive Medicine and Public Health,

School of Medicine, Keio University,

35 Shinanomachi, Sinjyuku-ku, Tokyo 160-8582, JAPAN

\section{Cover Letter}

The paper should be submitted with a covering letter stating

(1) that the paper is being submitted for consideration for publication in Environmental Health and Preventive Medicine;

(2) the exact address for all related correspondence to be sent to and telephone and FAX numbers of the author;

(3) that there has been no previous publication of the paper or a similar paper and that no such publication is intended;

(4) agreement of all authors as to content and authorship of paper;

(5) five key words describing the paper.

\section{Format and Style}

Manuscripts are to be written in clear, grammatical English. Papers that are not in Environmental Health and Preventive Medicine style or that are not in good idiomatic English will be returned to the author without review. Terminology and abbreviations not consistent with internationally accepted guidelines should be avoided. The manuscript should be typed on one side on ISO A4 paper with double spacing throughout, including in tables and illustration legends, with ample margins. Consecutive numbering of all pages is required, with the title page as pagel. The manuscript should follow the order: (a) title, (b) authors and complete names and locations of institutions or laboratories, (c) running title, (d) key words, (e) footnotes, (f) abstract, (g) text and references, (h) tables, (i) legends for all illustration, (j) illustrations and $(\mathrm{k})$ other material.

\section{Title Page}

Title

Titles should be informative and brief, and limited to about 100 characters. Key words necessary to identify the nature of the subject matter must be included in the title.

Authors and Their Affiliations

Authors must include their full names, complete with first and middle names or initials. Author's academic degrees should not be included. The full names of institutions and subsidiary laboratories must by given for each affiliated author together with current addresses, including postal code. The name, complete address, telephone and FAX number of the author to whom proofs and correspondence are to be sent should be included separately on the title page.

Running Title

A brief running title not to exceed 50 characters must be provided. Key Words

Five key words identifying the nature of the subject matter must be included on the title page. Footnotes to the title page text with superscript Arabic numerals are to be designated consecutively.

\section{Abstract}

The abstract should contain fewer than about 200 words yet be indicative of the content of the paper. It should indicate the purpose of the study, and the experimental technique, results, and interpretations of the date. All important terms relevant to the content of the paper should be incorporated into the abstract. Abbreviations should be kept to an absolute minimum; however, if they are needed, they must be properly identified so as to make the abstract independent of the text. 


\section{Introduction}

Brief reference to papers relevant to the problem or question which the author's investigation addresses are sufficient.

\section{Materials and Methods}

The methods should be briefly but clearly explained. Procedures published previously need only be cited in references. New and significant modifications need complete exposition. Papers reporting human experimentation must be in line with principles of the Helsinki Declaration of 1975 , as revised in 1983. Papers involving human subjects must include a statement that informed consent was obtained from each subject or subject's guardian.

Only results of those experiments in which proper attention has been given to experimental ethical considerations toward animals will be published. The statistical guidelines advocated by the International Committee of Medical Journal Editors (Ann Intern Med 1998 ; 108 : 266-73 )must be followed.

Results

Results should concisely summarize the findings of the study and follow the train of thought presented in the methods section. Textual description of the date must be presented in tables and illustrations.

\section{Discussion}

Discussion should emphasize the major findings of the study and its significance. Information presented in the previous section must not be repeated. Concise interpretation of date with wellfounded speculation may by included.

\section{Abbreviations for Scientific Units}

Abbreviations for Scientific Units must conform to the System International (SI units).

\section{Acknowledgments}

Acknowledgments should specify information on technical help, material and financial support including the source(s) and number(s) of the grant(s).

\section{References}

References should be numbered in the order of their first mention in the text ; cite only the number assigned to the reference. References should be double-spaced and be presented in numerical order. All references should be verified and checked that they have been cited in the text. Literature references must conform to the Uniform Requirements for Manuscripts Submitted to Biomedical Journals (JAMA 1993;269:2282-6) as the following examples.

For example:

(1) Papers published in Journals

Tarumi K, Hagihara A, Morimoto K. An investigation of effects lifestyle on care-seeking behavior using data from health insurance claims.Jpn J Hygiene 1995;49:984-97.

(2) Books and other monographs

Grundy F. Preventive Medicine and Public Health. London : H. K. Lewis Co. Ltd., 1960.

(3) Contributions in Books

Waldman LK. Multidimensional measurements of regional strains in the intact heart. In : Glass L, Hunter P, McCulloch A, editors. Theory of Heart. New York ; Springer-Verlag, 1991:145-74.

\section{Tables}

Tables of numerical data should be numbered and cited in numerical order in the text, and each be printed (also with doublespacing) on a separate page,numbered in sequence in Arabic numerals (Table 1, 2, etc.), provided with a heading, and referred to in the text as Table 1, Table 2,etc. Legends giving explanation sufficient to make the data intelligible without reference to the text must be typed on the same page.

\section{Illustrations and Illustration Legends}

Illustrations must be cited in the text in numerical order using Arabic numerals. Illustrations should be numbered in Arabic numerals according to the sequence of their appearance in the text, where they are referred to as Fig. 1, Fig. 2, etc. The name of the first author, the illustration number, and designation of the top of the illustration should be typed on a label adhered to the back of each illustration. Line drawings are to be submitted in either printouts from a laser printer or in camera-ready form on a white background, or in black ink. Illustration legends should be double-spaced on a separate sheet, and should include a brief title and a concise explanation of each illustration.

\section{Typesetting from Disks}

Environmental Health and Preventive Medicine is now copy editing accepted manuscripts electronically. Authors are encouraged to send a 3.5 inch floppy disk of the paper along with the required four hard copy printouts when submitting revised manuscripts. The entire manuscript should be contained in one file. Tables and mathematical material may be excluded from the disk file and presented only in the accompanying hard copy. Disks will not be returned to the authors. The disk should be labeled on the outside with Environmental Health and Preventive Medicine, the first author's name, a partial title of the manuscript, and the name of the computer file used to access the manuscript on disk. The name of the computer used (e.g., IBM/PS2), the name of the operating system and version (e.g. DOS3.3), and the word processing program and version (e.g. Word Perfect 5.0) must be provided. The following word processing programs are preferred: Word Perfect (IBM or Macintosh), Word perfect for Windows, Microsoft word (IBM or Macintosh), Mac Write $\lceil$. Authors should not use the Fast Save option on Macintosh computers. Ensure that the letter "l" and the number " 1 " (also " $O$ " and number " 0 " have been used properly, and format your article (tabs, indents, etc.) consistently. Characters not available on your word processor (Greek letters, mathematical symbols, etc.) should not be left open, but indicated by a unique code (e.g. @, \#, etc. for the Greek letters). Such codes must be used consistently throughout the text. The author should provide a list of such codes and a key. Do not allow your word processor to introduce word splits and do not use a 'justifed' layout. The Journal does not assume responsibility for errors in the conversion of newly released software, customized software, or special characters.

\section{Page Change and Reprints}

No page charge is necessary for black-and-white printing. In the case of color photographs the cost of publishing will be borne by the authors. Reprints may be ordered at prices shown in the reprint order form which will be sent to the author.

\section{Proofs}

Proofs will be sent to authors without manuscripts. Only printing errors should be corrected. Changes in the manuscript will not be allowed. The corrected proofs must be returned within 48 hours afterreceipt, preferably by FAX.

\section{Advertisements}

Advertisements must be received 5 weeks prior to the date of the issue in which the advertisement is to be published. Issues are published quarterly. Inquires regarding advertising should be directed to : Editorial Office, Department of Preventive Medicine and Public Health,

Human Research Inc.

2-5-16 Wakaba, Shinjyuku-ku, Tokyo 160-0011, Japan

TEL:+81(3) 3358-4001 FAX: +81(3) 3358-4002 